\title{
Aluminum doped silica preform fabrication using MCVD and solution doping technique: effects of various aluminum solution concentrations
}

\begin{abstract}
This work is described for solution doping in Modified Chemical Vapor Deposition (MCVD) used for silica optical fiber fabrication. This paper will concentrate on aluminum solution doping and the effect of different solution concentrations. The effect of three different concentrations of aluminum $(0.3 \mathrm{M}, 0.7 \mathrm{M}$ and $1.2 \mathrm{M})$ with the soot undergo heat treatment are studied while the other parameters of MCVD and solution doping are fixed such as deposition temperature, $\mathrm{SiCl} 4$ flow, and soaking time. The refractive index profile (RIP) of each doped preform is measured using preform analyzer to investigate aluminum distribution in the core region. Further investigation about $\mathrm{Al}$ distribution across the core sintered layer is also examined by EDX techniques.
\end{abstract}

Keyword: MCVD; Solution doping technique; Refractive index profile; EPMA (SEM-EDX) 